\title{
A Randomized, Single-Blinded Clinical Trial Evaluating the Effect of Extracorporeal Shockwave Treatment (ESWT) as Add-On Therapy to Vacuum Erectile Device on Peyronie's Disease
}

Jonas Mortensen'

Sune Møller Skov-Jeppesen ${ }^{1,2}$

Peter Bill Juul Ladegaard' Lars Lund ${ }^{1,2}$

'Department of Urology, Odense University Hospital, Odense, Denmark;

${ }^{2}$ Institute of Clinical Research, University of Southern Denmark, Odense, Denmark
Correspondence: Jonas Mortensen Department of Urology, Odense University Hospital, Sdr. Boulevard 29,

Odense, 5000, Denmark

Tel +4540172260

Email Jonas.Mortensen2@rsyd.dk
Purpose: The aim of this study was to investigate whether ESWT with a higher energy as an add-on therapy to vacuum-pump followed by manipulation exercises could reduce the penile curvature, pain and improve IIEF-5 score.

Materials and Methods: Men aged $>18$ and $<80$ years and diagnosed with PD in stable phase with no history of penile surgery or previous ESWT treatment were eligible to participate. They were randomised to either active ESWT $(n=16)$ or sham ESWT $(n=$ 16). Both groups were treated once a week for five weeks. Each treatment session consisted of 2000 shockwaves at $0.5 \mathrm{~mJ} / \mathrm{mm}^{2}$. All patients in both groups used a vacuum pump followed by manipulation exercises.

Results: Thirty men completed the study protocol. Mean age in the treatment group was 61.7 (SD 8.3) and 63 (SD 7.35) in the control group. After six months, mean change in penile curvature was -12.8 (SD 13) degrees in the treatment group and -6.6 (SD 8.9) in the control group $(\mathrm{p}=0.204)$. Mean IIEF-5 score decreased by 0.17 (SD 3) in the treatment group and 3.06 (SD 5.5) in the control group $(\mathrm{p}=0.086)$ at six-month follow-up. Pain was assessed using both VAS and PDQ and demonstrated no difference between the groups after six months $(\mathrm{p}=0.648)$.

Conclusion: In the treatment group, we observed a greater but non-significant change in penile curvature and no adverse effects.

Keywords: Peyronie's disease, ESWT, vacuum pump, erectile dysfunction, manipulation exercises

\section{Introduction}

Peyronie's disease (PD) is an acquired multifactorial fibrotic disorder of the tunica albuginea in the penile corpus cavernosum resulting in a palpable fibrous plaque. Plague formation may be accompanied by pain, penile curvature, plaque calcification, penile shortening and erectile dysfunction (ED). ${ }^{1}$ Some PD patients are unable to penetrate due to ED, painful erections and/or due to the angle of the curvature. ED is present in $20-50 \%$ of PD patients and occurs from a variety of reasons including penile curvature, performance anxiety and cavernosal fibrosis compromising the penile vascularity. ${ }^{2}$ Thus, PD may impact the patients' and their partners' quality of life significantly and increase the risk of depression, lower patients' self-esteem, and cause relationship difficulties. ${ }^{3}$ 
The epidemiological data on PD are limited and evidence regarding pathophysiology, prevalence and treatment is conflicting. The prevalence of PD was reported $3.2 \%$ in men aged $30-80$ years with the highest prevalence among men aged $60+$ years $(6.5 \%) .{ }^{4}$ Another study estimated the prevalence to be $8.9 \%$, and even higher in patients diagnosed with diabetes mellitus and hypertension. $^{5}$

The exact underlying pathogenesis behind PD is not fully understood, and the etiology remains unclear. However, many theories have been proposed, but none fully manage to comprehend the disease spectrum. ${ }^{6}$ A widely accepted thesis is that repeated microtraumas from e.g coitus in predisposed individuals result in fibrin deposition, penile curvature, chronic inflammation and plaque formation. The microtrauma theory attempts to justify PD as a wound-healing disorder. ${ }^{1,6}$ Furthermore, a strong association between Dupuytren's disease and PD has been found indicating a multifactorial history of the disease. ${ }^{5}$ Due to the incomplete understanding of the pathogenesis and heterogeneity of the disease, many different treatment strategies have been attempted.

Extracorporeal shockwave therapy (ESWT) has previously shown regenerative and wound-healing abilities in soft tissue. ${ }^{7}$ Thus, clinicians have started treating PD with ESWT.

The mechanism behind how ESWT affects the fibrous plaque is still unclear, but two hypotheses have been proposed. The first hypothesis suggests ESWT may work by directly damaging and remodelling the plaque. The second hypothesis argues that ESWT may increase the vascularisation by generating heat in the area, causing an inflammatory reaction with increased macrophage activity followed by plaque lysis and resorption. ${ }^{8}$

TGF- $\beta$ is an essential cytokine for creating a PD plaque and induces inflammation and chronic fibrosis. TGF- $\beta$ overexpression has been observed in PD plaques, ${ }^{1}$ and ESWT has shown the ability to reduce TGF- $\beta$ expression. ${ }^{9}$

A systematic review carried out in 2016 regarding ESWT treatment for PD concluded that ESWT might resolve pain, while the evidence that ESWT reduces curvature, plaque size and IIEF-score is inconclusive. ${ }^{10} \mathrm{An}$ improvement in penile curvature is considered as the gold standard when addressing treatment of PD.

The purpose of the present randomised safety study was to investigate the combined effect of ESWT with a high-energy level, vacuum pump, and manipulation exercises on Peyronie's disease.

\section{Materials and Methods}

\section{Inclusion}

This study was conducted in accordance with the Declaration of Helsinki. All patients were recruited from the outpatient clinic of the Department of Urology, Odense University Hospital (OUH), Denmark which has an uptake of patients residing in the region of Southern Denmark (1.2 million inhabitants). Recruitment took place from May 2018 until August 2019.

The inclusion criteria applied in this study were: Patients diagnosed with PD for more than 6 months and in stable phase, penile curvature 20-90 degrees, age 18-80 years, able to speak and understand Danish and provide written informed consent. Exclusion criteria were: Previous penile surgery and previous ESWT treatment. Stable disease phase was defined as no penile curvature change within the last 3 months or disease duration $>12$ months.

The study protocol was approved by the Danish Data Protection Agency and the Regional Ethics Committee (study ID: S-20170201). The study is registered at www. clinicaltrials.gov (study ID: NCT03530540).

\section{Randomization and Blinding}

To ensure valid randomisation, we used an in-house software (OPEN randomize) which was customized to create block randomization with a fixed block size unknown to the investigator. Block randomization was utilized due to the small sample size. Patients were randomised in 1:1 to either active ESWT or sham.

The investigator carried out the randomisation by entering the patient's unique social security number, which was then sent to an independent employee at the hospital who generated an ID for each patient and returned an email containing information regarding patient allocation.

Patients were blinded throughout the whole study period and group allocation was revealed after 6 months. The manufacturer (Storz Medical, Switzerland) provided gel pads. Sham pads were completely shockwave absorbent. During treatment, all settings and setups were identical in both the control and the treatment group. Curvature measurement was carried out blinded, and group allocation was concealed.

\section{Treatment}

Eligible patients were informed about the study design at the outpatient clinic before accepting an invitation to 
participate. No participants who were invited to participate refused. Those who orally accepted were subsequently contacted by telephone and invited to the first session. During the first session, patients provided written consent to participate and filled out questionnaires. All patients received thorough information on how to complete the questionnaires. A researcher was present at inclusion if patients had any uncertainty regarding specific questions in the questionnaires. Finally, patients were carefully introduced to manipulation exercises and vacuum pump therapy using the MANUAL Erection System (KESSEL Medintim GmbH, Morfelden-Walldorf, Germany). Calcification of the PD-plaque was assessed by ultrasonography by two investigators.

The instrument used to transmit the shockwaves was the DUOLITH SD1 manufactured by Storz Medical AG, Switzerland and owned by the Department of Urology. Treatment was conducted with two different stand-offs for either active or sham treatment. The stand-off applied to the sham was completely shockwave-absorbent. Besides using a different stand-off for the shockwave device, the settings and setup were identical in both groups to ensure that the patients were unable to recognize which treatment was being performed.

All patients received a total of five ESWT treatments once a week for five consecutive weeks. Both study groups were provided with a vacuum pump at baseline/inclusion date and directed to complete manipulation exercises daily for 10-15 minutes the next 6 months.

The patients started using vacuum pump and performing manipulation exercises after the first ESWT treatment. Patients were instructed to apply the vacuum pump to achieve a satisfactory erection and maintain in this position for 2 minutes. The vacuum was then released, and patients started to gently manipulate the erect penis for five minutes. Patients applied a constriction ring if they were unable to maintain an erection. Subsequently, the patients stretched the penis in flaccid state for 20 seconds and relaxed the tissue for 10 seconds. This process was repeated ten times.

Each patient received 2000 shockwaves targeting the plaque with energy ranging from $0.15 \mathrm{~mJ} / \mathrm{mm}^{2}$ to $0.5 \mathrm{~mJ} /$ $\mathrm{mm}^{2}$ at $3 \mathrm{~Hz}$ depending on the patient's pain threshold. Most patients did not sense any pain and received 2000 waves at a maximal energy of $0.5 \mathrm{~mJ} / \mathrm{mm}^{2}$. Patients who experienced pain were offered $200 \mathrm{mg}$ oral fentanyl (Lollipop). Adverse effects were systematically evaluated before each treatment in both groups.

\section{Outcomes}

To evaluate the participants, we used three questionnaires: International Index of Erectile Function (IIEF-5), Peyronie's Disease Questionnaire (PDQ) and the visual analogue scale (VAS). The IIEF-5 is composed of 5 questions regarding erectile function. Based on the patients' answers a final score was merged. The IIEF-5 score indicates the severity of ED: $5-7$ (severe), 8-11 (moderate), 12-16 (mild/moderate), 17-21 (mild), and 22-25 (no ED). All questionnaires have been internationally validated. ${ }^{1-14}$ Penile pain was quantified using VAS score, and the patients were asked about average pain sensation in the flaccid penis during the last 72 hours. The PDQ-questionnaire is designed to estimate the psychological and physical consequences (pain) of PD. We only applied the physical consequence for pain sensation.

All questionnaires were carefully introduced to each of the patients before completion to standardize all answers and avoid misunderstandings. The patients answered the questionnaires at baseline and at follow-up after one, three, and six months.

All patients submitted two photos of the erect penis used for measuring the penile curvature at baseline. The patients were instructed to take a photo from an angle that showed the maximum curvature of the penis. We compared the penile curvature on photographs taken by the patient at follow-up one, three, and six months after treatment was completed. All pictures and data were stored in Redcap. Penile curvature was measured with ImageJ (developed by the National Institutes of Health) using a standardized technique where two lines were applied to minimize inter- and intra-observer variations (Figure 1A and $\mathrm{B})$.

We conducted three follow-ups. At all follow-up visits, the patients reported; IIEF-5, VAS, PDQ-pain score, selfestimated effect, pictures displaying the penile curvature and how frequently the vacuum pump combined with manipulation exercises were utilized.

\section{Statistics}

Before initiating the study, a power analysis was carried out to estimate the sample size. We expected the mean difference between baseline and follow-up would increase by $100 \%$ in the treatment group compared to the control group. Thus, we ended up with a sample size of 32,16 in each arm, with $80 \%$ power and a statistical significance level of $5 \%$. 

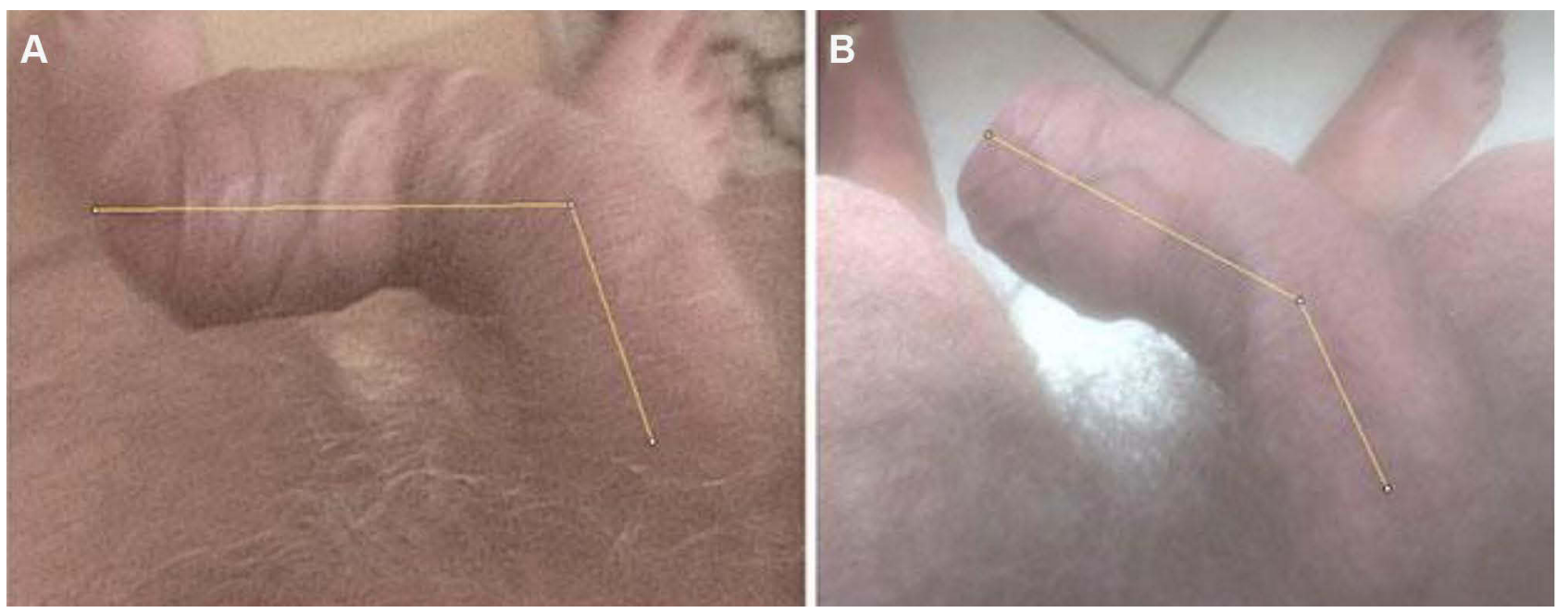

Figure I Demonstrating how we measured the penile curvature. Picture (A) is taken at inclusion, and picture (B) displays curvature at six months follow-up. Background: A 72-year old male who has suffered from PD for 12 months. He has previously been diagnosed with dupuytren's contracture. He has received ESWT for five consecutive weeks while applying the vacuum pump at home. His penile curvature improved 35 degrees. He reported moderate/high self-rated effect.

Baseline characteristics and follow-up data were compared between groups using $X^{2}$ (chi-square-test) and Mann-Whitney-U (MWU) test. $X^{2}$-test was applied to nominal, ordinal and binary data, whereas MWU was applied to numerical data (IBM ${ }^{\circledR}$ SPSS statistics software was used for data analysis).

Mean differences in IIEF-5, VAS, PDQ, and penilecurvature between the groups were compared using MWU. Wilcoxon Signed-Rank test was utilized for within-group penile curvature change.

\section{Results}

A total of 32 patients were randomised but only 30 completed the study protocol. Two patients in the treatment group suffered from phimosis and could not perform the exercises and were discarded.

We ended up with fourteen patients allocated to the treatment group and sixteen patients allocated to the control group. At 3 months follow-up, we had no dropouts. However, at six months we had 3: Two patients from the control group, and one patient from the treatment group. The patients who dropped out in the control group reported that they did not want to participate due to the lack of effect of the treatment, and they did not return questionnaires and photo. The patients from the treatment group had relapse of lymphoma.

In a sensitivity analysis, we used last observation carried forward for the patients who were lost to follow-up after six months. However, in the sensitivity analysis, the difference between the groups did not change significantly with respect to penile curvature, IIEF-5, or PDQ-pain compared to our primary analysis where statistical analysis was carried out with missing data for the patients who were lost to follow-up.

\section{Baseline Characteristics}

Baseline data is displayed in Table 1.

\section{Penile Curvature}

Penile curvature change is displayed in Figure 2. After six months, the mean change in penile curvature was -12.79 (SD 13) degrees in the treatment group, and -6.62 (SD $8.9)$ in the control group $(\mathrm{p}=0.204)$. At follow-up after one and three months the p-values were 0.317 and 0.560 , respectively. A within-group analysis was carried out displaying a statistically significant change in both the treatment $(p=0.004)$ and the control group $(p=0.017)$ from baseline to six months follow-up.

Calcified plaques were found in nine patients in the treatment group with 13.7 (SD 15.7) degrees mean change in penile curvature at 6 months follow-up. In the control group, calcified plaques were found in four patients with mean change of 14.5 (SD 6.35) degrees at 6 months follow-up. In patients with calcified plaques, there was no significant difference in penile curvature between the treatment group and the control group at six months follow-up $(\mathrm{p}=0.699)$.

Non-calcified plaques were found in five patients in the treatment group and in twelve patients in the control group. In the patients with non-calcified plaques, the mean change in penile curvature was 11.2 (SD 7.4) 
Table I Baseline Characteristics

\begin{tabular}{|c|c|c|c|}
\hline Variable & $\begin{array}{l}\text { Treatment } \\
\text { Group (SD) }\end{array}$ & $\begin{array}{l}\text { Control } \\
\text { Group (SD) }\end{array}$ & P-value \\
\hline Participants & 14 & 16 & \\
\hline Age, years & 6I.7I(8.3) & $63(7.35)$ & 0.83 \\
\hline BMI & $26.2(2.83)$ & $25.6(2.96)$ & 0.7 \\
\hline $\begin{array}{l}\text { Mean duration of PD } \\
\text { (months) }\end{array}$ & $16.21(14.1)$ & 18.38 (16.93) & 0.723 \\
\hline IIEF-5 & $19.5(4.1)$ & $18.75(3.84)$ & 0.692 \\
\hline VAS & $\mathrm{I} .86(2.7)$ & I.62 (2.45) & 0.814 \\
\hline Curvature (degrees) & $45(16.74)$ & $47.6(12.6)$ & 0.9 \\
\hline PDQ-pain score & $4.38(5.44)$ & $2.69(4)$ & 0.327 \\
\hline Smoking (pack years) & $10.29(14.34)$ & $16.1(16.4)$ & 0.334 \\
\hline $\begin{array}{l}\text { Alcohol consumption } \\
\text { (drinks/week) }\end{array}$ & & & 0.881 \\
\hline 0 & 3 & 2 & \\
\hline $0-7$ & 4 & 5 & \\
\hline $7-14$ & 2 & 2 & \\
\hline$|4-2|$ & 4 & 4 & \\
\hline $21+$ & I & 3 & \\
\hline $\begin{array}{l}\text { Dupuytren's } \\
\text { contracture }\end{array}$ & & & 0.28 \\
\hline Yes & 6 & 10 & \\
\hline No & 8 & 6 & \\
\hline $\begin{array}{l}\text { Sexual activity* } \\
\text { (times/month) }\end{array}$ & $4.86(2.54)$ & $5(3.52)$ & 0.883 \\
\hline Self-rated ED** & & & 0.151 \\
\hline Yes & 2 & 6 & \\
\hline No & 12 & 10 & \\
\hline Painful Erections & & & 0.765 \\
\hline Yes & 6 & 6 & \\
\hline No & 8 & 10 & \\
\hline $\begin{array}{l}\text { Calcified plaque on } \\
\text { ultrasound }\end{array}$ & & & $0.03^{*}$ \\
\hline Yes & 9 & 4 & \\
\hline No & 5 & 12 & \\
\hline $\begin{array}{l}\text { Charlson } \\
\text { comorbidity score }\end{array}$ & $2.36(1.5)$ & $1.94(0.68)$ & 0.552 \\
\hline
\end{tabular}

Notes: *Masturbation is considered as sexual activity; **ED, Erectile Dysfunction; All numbers are means; Plaque calcification is statistically different between groups.

degrees in the treatment group compared to 4 (SD 8.18) degrees in the control group at six months follow-up $(\mathrm{p}=0.138)$.

We observed a slight increase in penile curvature in seven patients; two patients in the treatment group and five patients in the control group. Penile curvature increased by an average of 5.5 degrees for the two patients in the

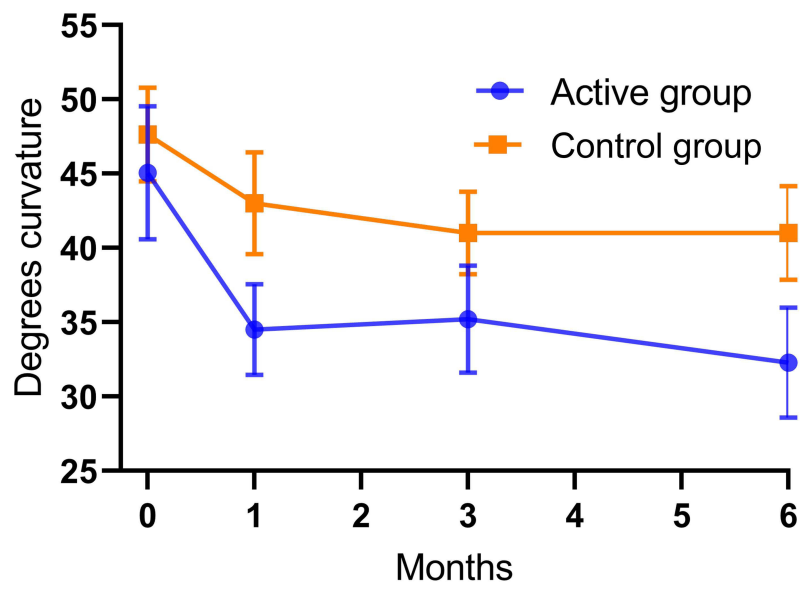

Figure 2 Displaying mean penile curvature and SEM at baseline, I month, 3 months and 6 months for both groups.

treatment group and by an average of 4 degrees for the five patients in the control group. However, only slight changes were observed, which might refer to varying picture quality that may have affected our measurement of penile curvature.

\section{IIEF-5 Score}

IIEF-5 change is displayed in Figure 3. Mean change in the treatment group was 0.17 (SD 3) compared to -3.06 (SD 5.5) in the control group at six-month follow-up $(\mathrm{p}=0.086)$. At follow-up at one and three months, the p-values were 0.369 and 0.058 .

\section{Pain}

Pain assessment was carried out using both VAS and PDQpain score. At six-month follow-up, VAS displayed increased pain in both groups with 0.92 (SD 2.1) in the

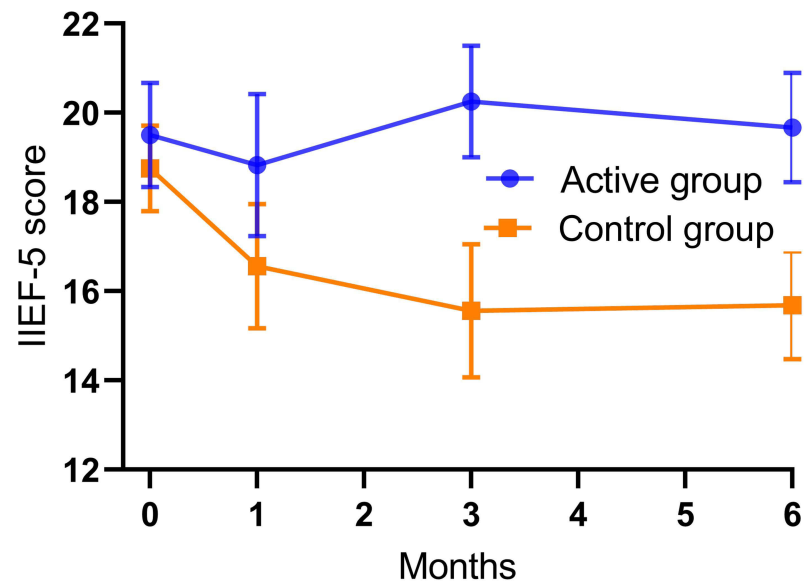

Figure 3 Displaying mean IIEF-5 and SEM at baseline, I month, 3 months and 6 months for both groups. 


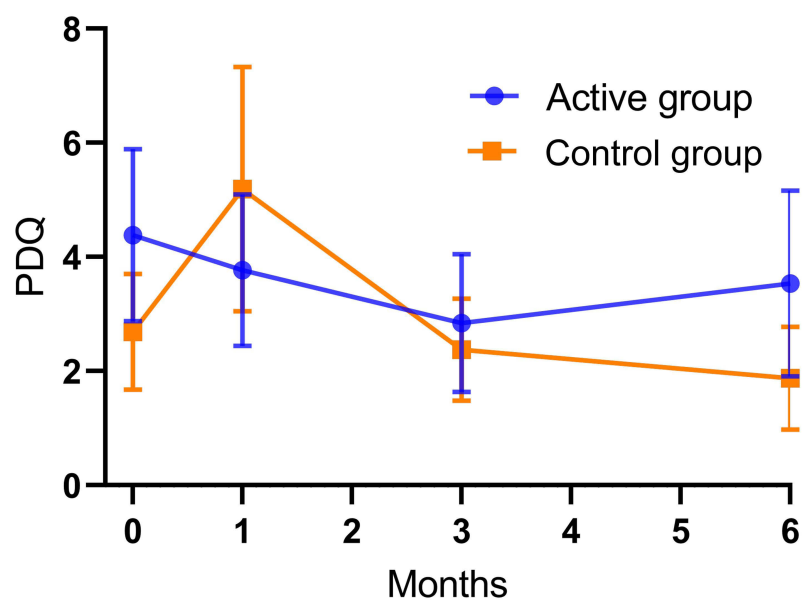

Figure 4 Displaying mean PDQ pain score and SEM at baseline, I month, 3 months and 6 months for both groups.

treatment group and 0.06 (SD 1.5) in the control group $(\mathrm{p}=0.772)$.

At follow-up at one and three months, the p-values were 0.94 and 0.96 .

PDQ-pain score decreased 0.85 (SD 7.3) in the treatment group and 0.81 (SD 2.5) in the control group after six months $(p=0.648)$. See Figure 4. At follow-up at one and three months, the p-values were 0.06 and 0.26 .

\section{Adverse Effect}

Twenty-nine out of 30 patients perceived little to no pain, while they were treated with ESWT. One patient in the treatment group required fentanyl during treatment. Twenty-nine out of 30 patients perceived little to no pain while applying the pump and performing the exercises. Twenty-eight out of 30 patients reported no pain $(<$ VAS 2) pain after manipulation exercises, two reported intense pain. At 6 months follow-up, the patients were asked if they felt any over-all improvement of their disease and mean usage of the vacuum pump. Results are displayed in Table 2.

\section{Discussion}

In this randomized clinical safety study, ESWT was combined with a vacuum pump and manipulation exercises. We observed a reduction of the penile curvature after six months by 12.79 degrees in the treatment group compared 6.62 degrees in the control group ( $p=0.204)$. IEEF-5 was unchanged in the treatment group in contrast to the control group where the score decreased six months after ESWT $(p=0.086)$.
Table 2 Self-Rated Effect 6 Months After Treatment Was Finished

\begin{tabular}{|l|l|l|l|}
\hline Group & $\begin{array}{l}\text { Treatment } \\
\text { Group* }\end{array}$ & $\begin{array}{l}\text { Control } \\
\text { Group }\end{array}$ & P value \\
\hline $\begin{array}{l}\text { Self-rated effect } \\
\text { Moderate/high }\end{array}$ & 3 & 0 & 0.117 \\
Low & 4 & 8 & \\
No effect & 7 & 8 & \\
\hline Vacuum pump and & 2.46 & 2.43 & 0.96 \\
manipulation exercises & & & \\
\hline
\end{tabular}

Notes: Self-reported mean usage (times/week) of vacuum pump and manipulation exercises is shown; *Only reported for 14 patients as two patients were discarded.

Pain was assessed using both VAS and PDQ but there were no significant differences between the groups. To our knowledge, no previous studies examined the add-on effect of vacuum pump to ESWT on PD. Vacuum pump and ESWT are both considered conservative treatments with few side effects. ${ }^{10,15}$

Continuous traction devices such as a penile vacuum pump employ its effect by increasing the activity in degrading enzymes. This initially causes loss of tissue strength and the PD plaque becomes soluble and degraded. The process is followed by an increase in newly synthesized collagen. ${ }^{8}$ We embedded manipulation exercises to further soften the plaque, thus enabling a larger potential for remodelling. The evidence supporting manipulation exercises is still very limited, although it has proven to be valuable when using Xiapex. ${ }^{16}$ A previous study demonstrated a significant decrease in penile curvature after 12 weeks $^{15}$ with usage of a vacuum pump for 10 minutes twice a day. A review argued that vacuum erectile devices are a viable option for managing PD, although more studies are needed to clarify the potential. ${ }^{17}$

In the present study, self-reported ED was found in $27 \%$ of our participants and was defined as lost ability to achieve a satisfactory erection in more than $50 \%$ of all attempts.

One way of quantifying ED is the IIEF-5 questionnaire. At baseline, we observed no statistical difference between the groups. However, at six months follow-up, the treatment group had a slightly increased score, while the control group had decreasing IIEF-5 score. ESWT has previously demonstrated an ability to improve vascularisation, wound healing, angiogenesis ${ }^{7}$ and $\mathrm{ED}^{18}$ which might explain why the IIEF-5 score increased in the treatment group. A meta-analysis ${ }^{18}$ revealed that ESWT could significantly increase IIEF-5 with energy flux set between 
0.09 and $0.25 \mathrm{~mJ} / \mathrm{mm}^{2}$. We applied ESWT with energy levels up to $0.5 \mathrm{~mJ} / \mathrm{mm}^{2}$ to maximize plaque remodelling. However, it is uncertain if vascularisation and angiogenesis increase at this energy level as well.

The decreased IIEF-5 score in the control group may be due to an initial overestimation - in both treatment groups. Several of our patients reported that they had been apprehensive about seeking treatment, and they had previously failed to acknowledge the actual impact of the disease. Repeatedly addressing the severity of their erectile dysfunction may have led the patients to submit a decreased, yet more precise IIEF-5 score at follow-up.

When addressing penile curvature, we observed a larger change in the treatment group compared to the control group but not statistically significant. However, patients in the treatment group clearly showed a higher degree of satisfaction compared to patients in the control group. In total, 50\% ( $22 \%$ moderate-high and $28 \%$ light improvement) of the patients in the treatment group reported subjective improvement of their disease, which seems clinically relevant.

We performed a secondary stratified analysis based on plaque calcification. We expected that calcified PD plaques were more likely to dissolve than non-calcified ones. However, in patients with calcified plaques, we found that the curvature reduction was comparable between the treatment group and the control group.

On the contrary, non-calcified plaques had a substantially higher curvature reduction when treated with active ESWT. In view of the overall result that showed no statistically significant benefit of ESWT on penile curvature, it has to be taken into consideration that there were more patients with calcified plaques in the treatment group, which potentially biased the result. Further studies need to take into account plaque calcification as a factor, which may influence the efficacy of ESWT.

Previous clinical studies have demonstrated a significant pain decrease among patients treated with ESWT using VAS. ${ }^{19,20}$ We applied both VAS and PDQpain. No significant changes were noticed between the groups. However, even though PDQ expands the assessment of pain, patients only reported a mean score of 4.38 and 2.69 in the treatment- and in the control group, respectively, at baseline. The low pain score may be explained by the inclusion of patients in stable disease phase with mean disease duration of 16.21 months in the treatment group and 18.38 months in the control group, and the fact that PD pain resolves spontaneously over time.
ESWT combined with a vacuum pump represents a previously unexplored combination. This study demonstrates shockwave therapy in combination with vacuum pump and manipulation exercise as a feasible and safe treatment for Peyronie's disease. We observed no clinical adverse events related to shockwave therapy applied with relatively high energy level.

\section{Side Effects}

We experienced no severe side effects to ESWT (bleeding, numbness, etc.). One patient was administered oral fentanyl before treatment with ESWT.

Regarding vacuum pump treatment followed by manipulation exercises, one patient in the control group experienced a small haematoma on the penile shaft. The haematoma resolved spontaneously and was most likely created by a very high negative pressure in the vacuum pump.

\section{Limitations}

Pictures of the erect penis were assessed with ImageJ by a single investigator. Measurements may fluctuate from time to time, although the procedure is standardized. Furthermore, patients should have used PDE-5 inhibitors before pictures were taken to ensure sufficient erection.

In a subanalysis, we demonstrated that the effect size of ESWT tended to be lower in patients with calcified plaques. We did not observe a significant change in penile curvature after ESWT, which might be explained by the fact that a relatively large proportion of the patients in the treatment group had calcified plaques.

\section{Conclusion}

We found no statistically significant change in any primary outcome. However, we observed a larger change in penile curvature in the treatment group and a greater subjective reported improvement. Furthermore, IIEF-5 remained stable in the treatment group, whereas IIEF-5 declined in the control group. Given that this study represents a noninvasive treatment combination with few side effects, more and larger randomised placebo controlled studies are needed to draw a final conclusion.

\section{Acknowledgments}

- The authors would like to thank KESSEL medintim who donated the vacuum pumps.

- Data storage is performed in REDCap via OPEN Odense Patient data Explorative Network, OUH 
Odense University Hospital. Furthermore, OPEN supplied data manager assistance related to the use of OPEN Randomise. OPEN's facilities are concurrent with applicable legislation on processing of special categories of personal data including health data.

- Study data were collected and managed using REDCap electronic data capture tools hosted at the Department of Urology, Odense University Hospital, Odense, Denmark. REDCap (Research Electronic Data Capture) is a secure, web-based software platform designed to support data capture for research studies, providing 1) an intuitive interface for validated data capture; 2) audit trails for tracking data manipulation and export procedures; 3 ) automated export procedures for seamless data downloads to common statistical packages; and 4) procedures for data integration and interoperability with external sources.

\section{Funding}

There is no funding to report.

\section{Disclosure}

The authors report no conflicts of interest in this work.

\section{References}

1. Campbell J, Alzubaidi R. Understanding the cellular basis and pathophysiology of Peyronie's disease to optimize treatment for erectile dysfunction. Transl Androl Urol. 2017;6(1):46-59. doi:10.21037/ tau.2016.11.01

2. Ostrowski KA, Gannon JR, Walsh TJ. A review of the epidemiology and treatment of Peyronie's disease. Res Rep Urol. 2016;8:61-70.

3. Ralph D, Gonzalez-Cadavid N, Mirone V, et al. The management of Peyronie's disease: evidence-based 2010 guidelines. J Sex Med. 2010;7(7):2359-2374. doi:10.1111/j.1743-6109.2010.01850.x

4. Schwarzer U, Sommer F, Klotz T, Braun M, Reifenrath B, Engelmann U. The prevalence of Peyronie's disease: results of a large survey. BJU Int. 2001;88(7):727-730. doi:10.1046/j.14644096.2001.02436.x

5. Mulhall JP, Creech SD, Boorjian SA, et al. Subjective and objective analysis of the prevalence of Peyronie's disease in a population of men presenting for prostate cancer screening. $J$ Urol. 2004;171(6 Part 1):2350-2353. doi:10.1097/01.ju.0000127744.18878.f1
6. Al-Thakafi S, Al-Hathal N. Peyronie's disease: a literature review on epidemiology, genetics, pathophysiology, diagnosis and work-up. Transl Androl Urol. 2016;5(3):280-289. doi:10.21037/tau.2016.04.05

7. Mittermayr R, Antonic V, Hartinger J, et al. Extracorporeal shock wave therapy (ESWT) for wound healing: technology, mechanisms, and clinical efficacy. Wound Repair Regen. 2012;20(4):456-465.

8. Hatzimouratidis K, Eardley I, Giuliano F, et al. EAU guidelines on penile curvature. Eur Urol. 2012;62(3):543-552. doi:10.1016/j. eururo.2012.05.040

9. Cui HS, Hong AR, Kim JB, et al. Extracorporeal shock wave therapy alters the expression of fibrosis-related molecules in fibroblast derived from human hypertrophic scar. Int J Mol Sci. 2018;19 (1):124. doi:10.3390/ijms19010124

10. Fojecki GL, Tiessen S, Osther PJS. Extracorporeal shock wave therapy (ESWT) in urology: a systematic review of outcome in Peyronie's disease, erectile dysfunction and chronic pelvic pain. World J Urol. 2017;35(1):1-9. doi:10.1007/s00345-016-1834-2

11. Rosen RC, Cappelleri JC, Gendrano N. The International Index of Erectile Function (IIEF): a state-of-the-science review. Int $J$ Impot Res. 2002;14:226. doi:10.1038/sj.ijir.3900857

12. Coyne Karin S, Currie Brooke M, Thompson Christine L, Smith Ted M. Responsiveness of the Peyronie's disease questionnaire (PDQ). J Sex Med. 2015;12(4):1072-1079. doi:10.1111/jsm.12838

13. Ferreira-Valente MA, Pais-Ribeiro JL, Jensen MP. Validity of four pain intensity rating scales. Pain. 2011;10:2399-2404.

14. Hellstrom WJG, Feldman R, Rosen RC, Smith T, Kaufman G, Tursi J. Bother and distress associated with Peyronie's disease: validation of the Peyronie's disease questionnaire. J Urol. 2013;190 (2):627-634. doi:10.1016/j.juro.2013.01.090

15. Raheem Amr A, Garaffa G, Raheem Tarek A, et al. The role of vacuum pump therapy to mechanically straighten the penis in Peyronie's disease. BJU Int. 2010;106(8):1178-1180. doi:10.1111/ j.1464-410X.2010.09365.x

16. Seftel AD. Re: safety and effectiveness of collagenase clostridium histolyticum in the treatment of Peyronie's disease using a new modified shortened protocol. J Urol. 2017;198(6):1193.

17. Avant RA, Ziegelmann M, Nehra A, Alom M, Kohler T, Trost L. Penile traction therapy and vacuum erection devices in Peyronie's disease. Sex Med Rev. 2019;7:338-348. doi:10.1016/j. sxmr.2018.02.005

18. Lu Z, Lin G, Reed-Maldonado A, Wang C, Lee YC, Lue TF. Lowintensity extracorporeal shock wave treatment improves erectile function: a systematic review and meta-analysis. Eur Urol. 2017;71 (2):223-233. doi:10.1016/j.eururo.2016.05.050

19. Hatzichristodoulou G, Meisner C, Gschwend Jürgen E, Stenzl A, Lahme S. Extracorporeal shock wave therapy in Peyronie's disease: results of a placebo-controlled, prospective, randomized, single-blind study. J Sex Med. 2013;10(11):2815-2821. doi:10.1111/jsm.12275

20. Palmieri A, Imbimbo C, Longo N, et al. A first prospective, randomized, double-blind, placebo-controlled clinical trial evaluating extracorporeal shock wave therapy for the treatment of Peyronie's disease. Eur Urol. 2009;56(2):363-370. doi:10.1016/j. eururo.2009.05.012
Research and Reports in Urology

\section{Publish your work in this journal}

Research and Reports in Urology is an international, peer-reviewed, open access journal publishing original research, reports, editorials, reviews and commentaries on all aspects of adult and pediatric urology in the clinic and laboratory including the following topics: Pathology, pathophysiology of urological disease; Investigation and treatment of urological disease; Pharmacology of drugs used for the treatment of urological disease. The manuscript management system is completely online and includes a very quick and fair peer-review system, which is all easy to use. Visit http://www.dovepress.com/ testimonials.php to read real quotes from published authors. 